\title{
MORBIDADES, CAPACIDADE FUNCIONAL E QUALIDADE DE VIDA DE MULHERES IDOSASa
}

\author{
Morbidity, functional capacity and quality of life of elderly women \\ La morbilidad, la capacidad funcional y calidad de vida de las mujeres mayores
}

Maurícia Brochado Oliveira Soares

Marina Aleixo Diniz ${ }^{4}$
Darlene Mara dos Santos Tavares ${ }^{2}$

Flávia Aparecida Dias ${ }^{3}$

\section{RESUMO}

Os objetivos foram comparar entre as faixas etárias: o estado conjugal, a escolaridade, a renda, o número de morbidades e de incapacidade funcional e os escores de qualidade de vida de mulheres idosas. Trata-se de um estudo de base populacional, transversal, realizado com 1.339 mulheres idosas. Utilizaram-se os instrumentos estruturados Older Americans Resources and Services, WHOQOL-BREF e WHOQOL-OLD. Para a comparação entre as variáveis categóricas foi utilizado o teste qui-quadrado e, para as numéricas, ANOVA-F, Bonferroni e Tamhane's de acordo com a homogeneidade das variâncias $(p<0,05)$. A comparação entre os grupos evidenciou que, quanto maior a faixa etária, menor a escolaridade e maior o número de incapacidade funcional. A maior faixa etária esteve associada a menores escores de qualidade de vida no domínio físico e facetas funcionamento dos sentidos e participação social. É mister analisar as características das mulheres idosas tendo em vista as especificidades nas diversas faixas etárias.

Palavras-chave: Enfermagem. Saúde da Mulher. Idoso. Envelhecimento.

\begin{abstract}
The objectives were to compare between the age groups: marital status, education, income, number of morbidities and functional disability scores of a quality of life from elderly women. This is a population-based study, sectional, applied to 1339 elderly women. We used the Older Americans Resources and Services structured instruments, WHOQOL-BREF and WHOQOL-OLD. To the comparison among the categorical variables we used chi-square for the numerical test, ANOVA-F, Tamhane and Bonferroni's according to the homogeneity of variances ( $p$ $<0.05$ ). The comparison among groups showed that the higher age, the lower is the educational level and larger number of functional disability. The highest age group was associated to lower scores of quality of life in the physical domain and operational facets of the senses and social participation. It is necessary to analyze the characteristics of elderly women considering the specificities of different ages.
\end{abstract}

Keywords: Nursing. Women's Health. Aged. Aging

\section{Resumen}

El objetivo fue comparar entre los grupos de edad: el estado civil, el educacional, el nivel de ingresos, el número de morbilidades y de discapacidad funcional y la calidad de vida de las mujeres de edad avanzada. Se trata de un estudio de base poblacional, transverso, realizado con 1.339 mujeres mayores. Se utilizaron instrumentos estructurados del Older Americans Resources and Services, WHOQOL-BREF y WHOQOL-OLD. Para comparar las variables categóricas se utilizó chi-cuadrado para la prueba numérica, ANOVA-F, Tamhane de Bonferroni y de acuerdo a la homogeneidad de varianzas $(p<0,05)$. La comparación entre grupos mostró que el nivel de edad mayor y menor nivel educacional aumentó el número de incapacidad funcional. El mayor grupo de edad se asoció con un menor desempeño de la calidad de vida en las facetas físicas y operativas de los sentidos y la participación social. Hay que analizar las características de las mujeres mayores, habida cuenta de la especificidad en diferentes grupos de edad.

Palabras clave: Enfermería. Salud de la Mujer. Anciano. Envejecimiento.

'Enfermeira Obstétrica. Mestranda em Atenção à Saúde do Programa de Pós-Graduação stricto sensu em Atenção à Saúde. Curso de Graduação em Enfermagem. Universidade Federal do Triângulo Mineiro (UFTM). Uberaba- MG. Brasil. Bolsista da CAPES/DS. E-mail: mauricia_olive@yahoo.com.br,'Enfermeira. Doutora em Enfermagem. Professora Associada do Curso de Graduação de Enfermagem. Departamento de Enfermagem e Educação em Saúde Comunitária da UFTM. Coordenadora do Programa de Pós-Graduação stricto sensu em Atenção à Saúde da UFTM. Uberaba-MG. Brasil. E-mail: darlenetavares@netsite.com.br, ${ }^{3}$ Enfermeira. Mestranda em Atenção à Saúde do Programa de Pós-Graduação stricto sensu em Atenção à Saúde. Curso de Graduação em Enfermagem da UFTM. Bolsista da CAPES/DS. Uberaba- MG. Brasil. E-mail: flaviadias_ura@yahoo.com.br., ${ }^{4}$ Enfermeira. Mestre em Atenção à Saúde. Professora Substituta do Curso de Graduação em Enfermagem. Departamento de Enfermagem e Educação em Saúde Comunitária da UFTM. Uberaba-MG. Brasil. E-mail: mafmtm@yahoo.com.br ,Terapeuta Ocupacional. Mestre em Ciências da Saúde. Terapeuta Ocupacional da UFTM. Uberaba- MG. Brasil. E-mail: sgeib@to.uftm.edu.br. 


\section{INTRODUÇÃO}

Em decorrência da interação dinâmica das taxas de fecundidade e mortalidade, observa-se o aumento na expectativa de vida da população. Com isso vem ocorrendo 0 envelhecimento populacional, que tem sido considerado um fenômeno mundial. No Brasil, em 1940, a expectativa média de vida do brasileiro era de 45,5 anos, já em 2008 passou para 72,8 anos. ${ }^{1}$

A população idosa passa por mudança na sua estrutura interna, tanto etária quanto na proporção entre os sexos. ${ }^{2}$ As mulheres vivem, aproximadamente, sete anos a mais do que os homens. $^{1}$

No município de Uberaba, Minas Gerais (MG), local do presente estudo, a população de mulheres idosas representa $57 \%$ do total de idosos. Esse percentual está acima dos encontrados para o Estado de MG (54,9\%) e para o Brasil $(55,4 \%){ }^{3}$ Tais dados reforçam a necessidade de desenvolver pesquisas direcionadas à população idosa feminina visando compreender o processo de feminização do envelhecimento.

Pesquisa realizada com o objetivo de identificar indicadores de saúde evidenciou que 30,8\% das mulheres idosas referem três ou mais doenças crônicas, 9,3\% estiveram acamadas nas últimas semanas e $14,1 \%$ interromperam suas atividades habituais nas últimas semanas devido aos problemas de saúde, ${ }^{4}$ fatores que, consequentemente, podem comprometer a qualidade de vida das mulheres idosas.

0 termo qualidade de vida possui vários conceitos, mas para fim deste estudo será adotado o da Organização Mundial de Saúde (OMS) que desenvolveu uma definição transcultural, considerada subjetiva, multidimensional, com aspectos positivos e negativos, a saber: percepção do indivíduo de sua posição na vida no contexto da cultura e sistema de valores nos quais ele vive e em relação aos seus objetivos, expectativas, padrões e

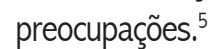

Entre as mulheres idosas alguns fatores podem contribuir para degenerar a qualidade de vida, entre eles a maior faixa etária quando acompanhada de limitações funcionais. ${ }^{6}$ Destaca-se que o status funcional interfere na realização das atividades da vida diária (AVD) quando associado às morbidades. ${ }^{7}$ Nesse contexto questiona-se a possível relação entre a faixa etária de mulheres idosas com suas características sociodemográficas, de saúde e sua qualidade de vida.

Desta forma, pesquisas que visam desvelar as características da população idosa feminina, evidenciando as suas especificidades, contribuem para o planejamento de ações em saúde norteado para a preservação da capacidade funcional e promoção da melhoria da qualidade de vida.

Este estudo objetivou comparar, entre as faixas etárias das mulheres idosas residentes no município de Uberaba/MG: 0 estado conjugal, a escolaridade, a renda, o número de morbidades, número de incapacidade funcional e os escores de qualidade de vida.

\section{MÉTODO}

0 presente estudo faz parte de um estudo maior, de base populacional, tipo inquérito domiciliar transversal, que avaliou a qualidade de vida de 2.143 idosos residentes na zona urbana do município de Uberaba/MG. 0 cálculo da amostragem populacional foi feito pelo Núcleo de Pesquisa em Saúde Coletiva da Universidade Federal do Triângulo Mineiro (UFTM), realizada em estudo anterior em que se descreveram as condições de saúde da população idosa. Consideraram-se $95 \%$ de confiança, $80 \%$ de poder do teste, margem de erro de $4,0 \%$ para as estimativas intervalares e uma proporção estimada de $p=0,5$ para as proporções de interesse. Para a seleção dos idosos utilizou-se a técnica de amostragem estratificada proporcional levando-se em consideração os diversos bairros como estratos.

Para a condução da presente investigação utilizaramse os seguintes critérios de inclusão: ser do sexo feminino, ter 60 anos ou mais de idade, pontuação mínima de 13 pontos na avaliação cognitiva, morar na zona urbana da cidade de Uberaba/MG e concordar em participar do estudo. Um total de 1.339 mulheres idosas preencheu os critérios de inclusão.

A avaliação cognitiva foi baseada no Miniexame do Estado Mental (MEEM), versão reduzida validada pelos pesquisadores do Projeto SABE. ${ }^{8}$ Nesta versão estabeleceu-se o ponto de corte de 12/13, obtendo-se uma sensibilidade de $93,8 \%$ e especificidade de 93,9\%. A deterioração cognitiva foi indicada por pontuação igual ou inferior a $12 .{ }^{8}$

0 instrumento estruturado foi baseado no questionário Older Americans Resources and Services (OARS), elaborado pela Duke University e adaptado à realidade brasileira, que consta dos seguintes itens: dados sociodemográficos e econômicos, AVD e saúde. ${ }^{9}$ Foi considerada incapacidade funcional quando o idoso referiu não conseguir realizar a AVD sem auxílio. 0 número de incapacidade funcional foi obtido por meio da soma das AVDs que os idosos não conseguiam realizar. Para a avaliação da qualidade de vida utilizaram-se os instrumentos WHOQOL-BREF eWHOQOLOLD, validados no Brasil. ${ }^{10,11}$

As variáveis estudadas foram: faixa etária (60-70, $70-80,80$ anos ou mais), estado conjugal (casada ou mora com companheiro; separada/desquitada/divorciada, viúva e solteira), escolaridade em anos de estudo (sem escolaridade; $1-4 ; 4-8 ; 8 ; 9-11$ e 11 ou mais), renda individual, em salários mínimos (sem renda; $<1 ; 1 ; 1-3 ; 3-$ $5 ; 5$ ou mais); número de morbidades ( $0 ; 1-3 ; 3-6 ; 6-9 ; 9-$ $12 ; 12$ ou mais); número de incapacidade funcional ( $0 ; 1$ 3; 3 ou mais); qualidade de vida mensurada pelo WHOQOLBREF (domínios: físico, psicológico, relações sociais e meio ambiente) e WHOQOL-OLD (facetas: funcionamento dos sentidos, autonomia, atividades passadas, presentes e futuras, participação social, morte, morrer e intimidade).

Optou-se pela entrevista direta na aplicação dos instrumentos de qualidade de vida, que podem ser 
autoaplicáveis, em razão da possível dificuldade de leitura, problemas visuais e analfabetismo entre os idosos. As perguntas foram respondidas tendo como base as duas últimas semanas de vida. Os entrevistadores foram capacitados antes de iniciar a pesquisa e acompanhados pelos supervisores de campo.

Foi construída planilha eletrônica no programa Excelß, e os dados coletados foram digitados, em dupla entrada. Posteriormente, foi verificada a consistência entre os campos; quando inconsistentes, retornou-se a entrevista original para correção.

Os dados foram submetidos à análise descritiva por meio das frequências simples absolutas e percentuais. Cada domínio do WHOQOL-BREF e facetas do WHOQOL-OLD foram analisados isoladamente, tabulados e consolidados no software SPSS, com suas referidas sintaxes. Para a comparação entre as variáveis categóricas foi utilizado o teste qui-quadrado e, para as numéricas, ANOVA-F seguida pelo teste de Bonferroni ou Tamhane's de acordo com a homogeneidade das variâncias. Os testes foram considerados significativos quando $p<0,05$.

Esta pesquisa foi aprovada pelo Comitê de Ética em Pesquisa com Seres Humanos da UFTM, protocolo No 897. Os idosos foram contactados em seus domicílios, e, somente após a anuência do entrevistado e assinatura do Termo de Consentimento Livre e Esclarecido conduziu-se a entrevista.

\section{RESULTADOS E DISCUSSÃO}

A distribuição das frequências das variáveis estudadas em relação à faixa etária é mostrada na Tabela 1.

Tabela 1. Distribuição de frequência das características sociodemográficas, número de incapacidade funcional e de morbidades segundo faixa etária das idosas. Uberaba, 2010. n=1.339

\begin{tabular}{|c|c|c|c|c|c|c|c|c|c|}
\hline \multirow{2}{*}{$\begin{array}{l}\text { Faixa etária } \\
\text { Variáveis }\end{array}$} & \multicolumn{2}{|c|}{$60-70$} & \multicolumn{2}{|c|}{$70-80$} & \multicolumn{2}{|c|}{$\begin{array}{l}80 \text { ou } \\
\text { mais }\end{array}$} & \multicolumn{2}{|c|}{ Total } & \multirow[b]{2}{*}{$p$} \\
\hline & $\mathrm{n}$ & $\%$ & $\mathrm{n}$ & $\%$ & $\mathrm{n}$ & $\%$ & $\mathrm{n}$ & $\%$ & \\
\hline \multicolumn{10}{|l|}{$\overline{\text { Estado conjugal }}$} \\
\hline Casada/mora com companheiro & 293 & 47,7 & 173 & 33,3 & 30 & 14,6 & 496 & 37,0 & \multirow{4}{*}{$<0,001$} \\
\hline Separada/d esquitada/divorciada & 78 & 12,7 & 39 & 7,5 & 3 & 1,5 & 120 & 9,0 & \\
\hline Viúva & 198 & 32,2 & 289 & 55,7 & 160 & 78,0 & 647 & 48,3 & \\
\hline Solteira & 45 & 7,3 & 18 & 3,5 & 12 & 5,9 & 75 & 5,6 & \\
\hline \multicolumn{10}{|l|}{ Escolaridade (em anos) } \\
\hline Sem escolaridade & 104 & 17,0 & 117 & 22,6 & 58 & 29,3 & 279 & 20,8 & \multirow{6}{*}{$<0,001$} \\
\hline $1-4$ & 200 & 32,8 & 181 & 34,9 & 57 & 28,8 & 438 & 32,7 & \\
\hline 4- 8 & 210 & 34,4 & 169 & 32,6 & 57 & 28,8 & 436 & 32,6 & \\
\hline 8 & 23 & 3,8 & 25 & 4,8 & 5 & 2,5 & 53 & 4,0 & \\
\hline $9-11$ & 16 & 2,6 & 1 & 0,2 & 9 & 4,5 & 26 & 1,9 & \\
\hline 11 ou mais & 57 & 9,3 & 25 & 4,8 & 12 & 6,1 & 94 & 7,0 & \\
\hline \multicolumn{10}{|l|}{$\begin{array}{l}\text { Renda individual (em salário } \\
\text { mínimo)* }\end{array}$} \\
\hline Sem renda & 132 & 21,7 & 46 & 8,9 & 14 & 6,8 & 192 & 14,3 & \multirow{6}{*}{$<0,001$} \\
\hline$<1$ & 16 & 2,6 & 3 & 0,6 & 1 & 0,5 & 20 & 1,5 & \\
\hline 1 & 331 & 54,4 & 333 & 64,5 & 117 & 57,1 & 781 & 58,3 & \\
\hline $1-3$ & 104 & 17,1 & 112 & 21,7 & 63 & 30,7 & 279 & 20,8 & \\
\hline $3-5$ & 16 & 2,6 & 17 & 3,3 & 9 & 4,4 & 42 & 3,1 & \\
\hline$>5$ & 9 & 1,5 & 5 & 1,0 & 1 & 0,5 & 15 & 1,1 & \\
\hline \multicolumn{10}{|l|}{ Número de morbidades } \\
\hline Nenhum & 11 & 1,8 & 3 & 0,6 & - & - & 14 & 1,0 & \multirow{6}{*}{0,187} \\
\hline $1-3$ & 58 & 9,4 & 39 & 7,5 & 15 & 7,3 & 112 & 8,4 & \\
\hline $3-6$ & 169 & 27,5 & 141 & 27,1 & 46 & 22,4 & 356 & 26,6 & \\
\hline $6-9$ & 195 & 31,8 & 184 & 35,4 & 74 & 36,1 & 453 & 33,8 & \\
\hline $9-12$ & 133 & 21,7 & 106 & 20,4 & 47 & 22,9 & 286 & 21,4 & \\
\hline 12 ou mais & 48 & 7,8 & 47 & 9,0 & 23 & 11,2 & 118 & 8,8 & \\
\hline \multicolumn{10}{|l|}{$\begin{array}{l}\text { Número de incapacidades } \\
\text { funcionais }\end{array}$} \\
\hline Nenhuma & 506 & 82,4 & 342 & 65,8 & 79 & 38,5 & 927 & 69,2 & \multirow{3}{*}{$<0,001$} \\
\hline $1-3$ & 95 & 15,5 & 151 & 29,0 & 102 & 49,8 & 348 & 26,0 & \\
\hline 3 ou mais & 13 & 2,1 & 27 & 5,2 & 24 & 11,7 & 64 & 4,8 & \\
\hline
\end{tabular}


A comparação entre os grupos evidenciou que, entre as mulheres idosas de 60-70 anos, predominaram as casadas ou que moram com companheiro, enquanto as de $70-80$ e 80 ou mais são viúvas $\left(\div^{2}=155,821 ; p<0,001\right)$. Em estudo com mulheres idosas observou-se que $44,7 \%$ eram viúvas. ${ }^{12}$ Este fato pode estar relacionado à maior longevidade do sexo feminino evidenciando a perda do companheiro no decorrer dos anos. A falta do companheiro, entre as mulheres idosas longevas, pode ser um fator que predispõe aos sentimentos de solidão e tristeza, gerando a desmotivação para o autocuidado. A enfermagem pode identificar tais fatores visando desenvolver estratégias que motivem a inserção e participação social das mulheres idosas, de forma a amenizar os sentimentos negativos pela ausência de companheiro.

Observou-se que, quanto maior a faixa etária menor a escolaridade, predominando entre as mulheres idosas de 60-70 anos 8 anos de estudo, entre aquelas com 70-80 1-4 anos e de 80 ou mais, 0 analfabetismo $\left(\div^{2}=41,562 ; p<0,001\right)$. Em outra pesquisa conduzida com idosas de baixa renda prevaleceu 0 analfabetismo (49,3\%), independente da faixa etária, com percentuais superiores ao encontrado no presente estudo. ${ }^{12}$

É possível que o maior grau de escolaridade entre as idosas mais jovens tenha relação com os avanços ocorridos nos níveis de educação da população brasileira. Entre 1940 e 2000 houve um aumento significativo na proporção de idosos alfabetizados. ${ }^{13} \mathrm{O}$ avanços nos parâmetros educacionais entre idosos contribuem para a melhoria das condições de saúde, na medida em que a compreensão sobre a ação educativa favorece o aumento à adesão ao tratamento, a realização de medidas preventivas de doenças e promocionais de saúde. Nesta perspectiva, a enfermagem deve adequar as estratégias das ações em saúde à escolaridade das mulheres idosas.

Entre as mulheres idosas que não apresentaram renda, observa-se um predomínio daquelas com 60-70 anos. Contudo, em todas as faixas etárias estudadas evidencia-se maior frequência de mulheres idosas com renda estimada em um salário mínimo. Destaca-se que, entre as mulheres idosas que recebem 1-3 salários predominam as com 80 anos ou mais $\left(\div^{2}=72,630 ; p<0,001\right)$. A baixa renda foi evidenciada em outro estudo, no qual $62,3 \%$ das mulheres idosas recebiam até um salário mínimo e $22 \%$ não possuíam renda. ${ }^{12}$ Embora este aspecto não tenha sido investigado neste estudo, o fato de as mulheres idosas de 60-70 anos não possuírem renda pode estar relacionado ao estado conjugal, pois a maioria é casada e pode depender financeiramente do marido. Por outro lado, nas maiores faixas etárias a maioria é viúva e a renda pode ser proveniente de pensão.

Mais de $98 \%$ das mulheres idosas de todas as faixas etárias apresentaram pelo menos uma morbidade. Não se observou diferença significativa na frequência do número de morbidades entre as faixas etárias estudadas $\left(\div^{2}=13,703\right.$; $p=0,187)$. Investigação com base na Pesquisa por Amostra Nacional de Domicílios (PNAD) evidenciou que, independente da faixa etária, houve prevalência de três ou mais morbidades entre as mulheres idosas. ${ }^{4}$

Destaca-se que as mulheres idosas de 80 anos ou mais referiu pelo menos uma morbidade. Inquérito realizado com mulheres idosas nesta faixa etária apresentou maior prevalência de doenças, corroborando os dados da presente pesquisa. ${ }^{7}$ Neste estudo, verificou-se que as mulheres idosas com 80 anos ou mais possuíam, em sua maioria, 1 - 8 anos de estudo, fato este que pode favorecer a presença do maior número de morbidades. 0 acesso aos serviços de saúde, bem como às informaç̃̃es, pode ser limitado em decorrência da baixa escolaridade. Neste contexto, é mister a investigação da escolaridade entre as idosas visando planejar estratégias para o cuidado em saúde, de forma que favoreça a promoção do autocuidado e a adesão ao tratamento.

Observou-se que a maioria das mulheres idosas de 6070 anos e 70-80 não tem incapacidades funcionais, enquanto nas com 80 ou mais prevaleceu $1-3$ incapacidades $\left(\div^{2}=146,624\right.$; $p<0,001)$. Além disso, a proporção de eventos aumenta progressivamente à medida em que aidade aumenta. Investigação conduzida com base na PNAD observou que, conforme aumentou a faixa etária, aumentou a prevalência de incapacidade funcional entre as mulheres, ${ }^{4}$ corroborando os dados desta pesquisa.

Inquérito realizado com mulheres idosas identificou que aquelas com 80 anos ou mais apresentam maior chance de ter limitações funcionais $(O R=5,99)$, o que pode anteceder a condição de incapacidade funcional. A identificação de fatores relacionados com as limitações funcionais permite a elaboração de políticas públicas direcionadas à preservação da autonomia da mulher idosa. ${ }^{6}$ Pesquisa realizada com idosos verificou que a prevalência de incapacidade funcional é significativamente superior entre aqueles com idade maior ou igual a 70 anos $(p<0,001)$ e sexo feminino $(p<0,002) .^{14}$

A investigação sobre a capacidade de realizar as AVDs não tem sido incluída rotineiramente no cuidado ao idoso na Atenção Primária. Neste contexto, a enfermagem pode acrescentar esta informação nos dados coletados durante a consulta de enfermagem e visita domiciliar. Por outro lado, as instituições de ensino devem capacitar os futuros profissionais de saúde para identificar, precocemente, as limitações funcionais na mulher idosa.

A capacidade funcional é fundamental para 0 desempenho das atividades cotidianas, sendo necessária aos serviços e para que os profissionais de saúde realizem ações que estimulem a preservação e a manutenção da funcionalidade da mulher idosa. Desta forma, estará contribuindo para postergar a dependência, que demanda maior atenção dos serviços de saúde e acarreta prejuízos para a mulher idosa, a família e a sociedade.

Na Tabela 2 apresenta-se a distribuição dos escores de qualidade de vida mensurados pelos instrumentos WHOQOLBREF e WHOQOL-OLD segundo as faixas etárias. 
Tabela 2. Distribuição dos escores de qualidade de vida, WHOQOL-BREF e WHOQOL-OLD, segundo faixa etária. Uberaba, 2010. $n=1.339$

\begin{tabular}{|c|c|c|c|c|c|c|c|c|c|}
\hline \multirow{2}{*}{$\frac{\text { Faixa etária }}{\text { Escore de qualidade de vida }}$} & \multicolumn{2}{|c|}{$60-70$} & \multicolumn{2}{|c|}{$70-80$} & \multicolumn{2}{|c|}{80 ou mais } & \multicolumn{2}{|c|}{ Total } & \multirow[b]{2}{*}{$p$} \\
\hline & Média & $\begin{array}{l}\text { Desvio- } \\
\text { padrão }\end{array}$ & Média & $\begin{array}{c}\text { Desvio- } \\
\text { padrão }\end{array}$ & Média & $\begin{array}{l}\text { Desvio- } \\
\text { padrão }\end{array}$ & Média & $\begin{array}{l}\text { Desvio- } \\
\text { padrão }\end{array}$ & \\
\hline \multicolumn{10}{|l|}{ WHOQOL-BREF } \\
\hline Físico & 60,07 & 15,97 & 58,75 & 16,01 & 53,88 & 17,26 & 58,61 & 16,31 & $<0,001$ \\
\hline Psicológico & 64,94 & 13,16 & 66,32 & 12,26 & 64,65 & 12,23 & 65,43 & 12,69 & 0,121 \\
\hline Social & 68,18 & 12,9 & 69,22 & 11,67 & 67,76 & 10,74 & 68,52 & 12,13 & 0,221 \\
\hline Meio ambiente & 61,17 & 13,48 & 62,68 & 11,26 & 63,27 & 10,68 & 62,08 & 12,27 & 0,39 \\
\hline \multicolumn{10}{|l|}{ WHOQOL-OLD } \\
\hline Funcionamento dos sentidos & 81,58 & 18,68 & 79,55 & 19,68 & 75,61 & 22,18 & 79,88 & 19,73 & 0,001 \\
\hline Autonomia & 60,28 & 14,08 & 60,16 & 12,79 & 58,73 & 12,87 & 59,99 & 13,41 & 0,338 \\
\hline Atividades pas., pres. e futuras & 64,42 & 13,61 & 66,66 & 12,09 & 66,02 & 10,87 & 65,54 & 13,18 & 0,015 \\
\hline Participação social & 64,9 & 14,96 & 65,37 & 14,97 & 61,64 & 16,53 & 64,59 & 15,26 & 0,01 \\
\hline Morte e morrer & 73,78 & 25,63 & 74,34 & 25,12 & 76,74 & 23,88 & 74,45 & 25,17 & 0,344 \\
\hline Intimidade & 67,36 & 17,96 & 69,01 & 15,95 & 68,81 & 15,53 & 68,22 & 16,85 & 0,221 \\
\hline
\end{tabular}

A comparação entre os grupos etários evidenciou que, quanto maior a faixa etária, menor o escore de qualidade de vida referente ao domínio físico ( $\mathrm{F}=11,272 ; p<0,001)$. Inquérito de base populacional realizado com idosos utilizando o instrumento genérico de avaliação de qualidade de vida Short Form (SF)-36 demonstrou que, quanto maior a faixa etária, menores os escores de qualidade de vida em todos os seus domínios, a saber, capacidade funcional; aspectos físicos; dor; estado geral da saúde; vitalidade; aspectos sociais, aspectos emocionais e saúde mental..$^{15}$

0 domínio físico avalia o impacto da dor e desconforto, energia e fadiga, sono e repouso, mobilidade, atividades da vida cotidiana, dependência de medicação ou de tratamentos e capacidade de trabalho na qualidade de vida. ${ }^{10}$ Cabe ressaltar que, apesar de o número de morbidade não apresentar diferença significativa entre as faixas etária, na Tabela 1, observa-se que, entre as idosas com 80 anos ou mais, ocorre um aumento percentual na maior ocorrência de 9-12 e 12 ou mais morbidades em relação às outras faixas etárias. Ademais, as mulheres idosas com 80 anos ou mais apresentam maior número de incapacidade funcional para a realização das atividades da vida diária. Estes fatos podem estar impactando de forma negativa no domínio físico da qualidade de vida da mulher idosa com 80 anos ou mais.

Em geral, nos serviços de saúde, investigam-se as morbidades presentes. Contudo, por vezes, não se leva em consideração o impacto das polimorbidades no planejamento do cuidado de enfermagem. Nesta perspectiva, é necessário utilizar os dados de morbidades para elaborar o cuidado de enfermagem tendo como enfoque a promoção do autocuidado, da autonomia e da inserção social e, consequentemente, a melhoria da qualidade de vida.

Nos demais domínios de qualidade de vida, a saber, psicológico, social e meio ambiente, não foram observadas diferenças significativas entre as faixas etárias.

Concernente à qualidade de vida mensurada pelo WHOQOL-OLD, verificou-se que, conforme aumentou a faixa etária, ocorreu diminuição do escore relacionado à faceta funcionamento dos sentidos ( $\mathrm{F}=7,173 ; p=0,001)$, divergente do encontrado em estudo realizado com idosos participantes de grupos de terceira idade utilizando o mesmo instrumento de qualidade de vida desta pesquisa, o qual não observou diferenças significativas nesta faceta em relação às faixas etárias. $^{16}$

A faceta funcionamento dos sentidos avalia as perdas na audição, paladar, visão, tato e olfato com possíveis repercussões na vida diária, interferência na participação em atividades familiares e sociais e na habilidade de interagir com outras pessoas. ${ }^{11}$ As perdas das habilidades sensoriais podem impactar negativamente na autoestima e no autocuidado à saúde, refletindo, assim, na piora da qualidade de vida das mulheres idosas. Detectando precocemente estas perdas e as potencialidades das mulheres idosas, a enfermagem poderá auxiliá-las, bem como aos seus familiares, visando diminuir os riscos físicos e estimular a interação social.

$\mathrm{Na}$ faceta atividades passadas, presentes e futuras, as mulheres idosas de 60-70 anos apresentaram escore significativamente inferior comparadas às outras faixas etárias ( $\mathrm{F}=4,234 ; p=0,015)$. Resultado divergente ao encontrado em inquérito com idosos participativos de grupos de terceira idade que não observou diferença significativa nos escores de qualidade de vida, WHOQOL-OLD, entre as faixas etárias. ${ }^{16}$ Esta faceta descreve a satisfação com as conquistas ocorridas no decorrer da vida e com objetivos a que se anseia. ${ }^{11}$ Ao analisar as características sociodemográficas das mulheres idosas deste estudo, verificou-se que aquelas com 60-70 anos possuem menor renda em relação às demais faixas etárias. A menor renda, ou falta desta, é um fator limitante na autonomia para aquisição de bens materiais. Contudo, há de se investigar outros fatores que interferem na satisfação com as conquistas da vida, bem como com suas aspirações não relacionadas aos aspectos materiais. 
Na faceta participação social, as mulheres idosas de 80 anos e mais apresentaram menor escore em relação às demais faixas etárias ( $\mathrm{F}=4,643 ; p=0,01)$. Destaca-se que, entre as mulheres idosas com 70-80 anos, os escores são maiores do que as que estão com 60-70 anos. Divergente destes resultados, pesquisa realizada com idosos que participavam de grupos de terceira idade utilizando o WHOQOLOLD não observou diferenças significativas nesta faceta em relação às faixas etárias. ${ }^{16}$

As mulheres idosas com 80 anos e mais possuem maior número de morbidades e de incapacidade funcional. Tais fatores podem estar restringindo a participação das mulheres idosas em atividades na comunidade, dadas as limitações impostas pela doença, bem como a maior dependência de terceiros, interferindo, assim, negativamente na qualidade de vida.

Observa-se que determinados domínios e facetas da qualidade de vida estão relacionados com certas características apresentadas pelas mulheres idosas que compõem as faixas etárias. A enfermagem deve identificar estas especificidades visando desenvolver estratégias de enfrentamento com o apoio da mulher idosa, familiares, cuidadores e comunidade. Assim, será possível realizar 0 atendimento de acordo com as necessidades das mulheres idosas, fortalecendo a atenção qualificada da profissão e contribuindo para a melhoria das condições de saúde e qualidade de vida desta população.

\section{CONCLUSÃO}

0 maior número de incapacidades funcionais relacionado à maior faixa etária evidenciou a necessidade de ações em saúde preventivos e promocionais. Deve-se enfatizar nos serviços de saúde a identificação das dificuldades vivenciadas pelas mulheres idosas e buscar alternativas que estimulem a funcionalidade postergando as limitações. Os menores escores de qualidade de vida entre as mulheres idosas mais velhas remetem à necessidade de maior atenção a esta população em busca de proporcionar melhoria aos aspectos físicos, minimização do impacto do funcionamento sensorial por meio de medidas adaptativas e desenvolvimento e estímulo à participação social.

Os resultados deste trabalho evidenciaram que é mister analisar as características sociodemográficos e de saúde das mulheres idosas, tendo em vista as especificidades destes grupos considerando a variabilidade das condições de saúde e qualidade de vida nas diversas faixas etárias. É necessário que os serviços e profissionais de saúde considerem a multidimensionalidade e peculiaridades do contexto de vida das mulheres idosas, visando a manutenção da independência, autonomia, inserção social e melhoria da qualidade de vida.

Neste estudo, as morbidades foram autorreferidas; desta forma, podem estar subestimadas em decorrência do desconhecimento das mulheres idosas, constituindo-se em uma limitação deste estudo.

\section{REFERÊNCIAS}

1.Instituto Brasileiro de Geografia e Estatística-IBGE. Projeção da população do Brasil por sexo e idade para o período 1980-2050: revisão 2008. [Internet]. [citado 2010 jan 7]. Disponível em: http:// www.ibge.gov.br.

2.Carvalho JAM, Rodrigues-Wong LL. A transição da estrutura etária da população brasileira na primeira metade do século XXI. Cad. Saude Publica. 2008; 24(3): 597-605.

3.Ministério da Saúde (BR).Departamento de Informática do SUS. Caderno de Informações de Saúde. [Internet]. [citado 2010 jan 5]. Disponível em: http://tabnet.datasus.gov.br/tabdata/cadernos/ cadernosmap.htm .

4.Lima-Costa MF, Loyola Filho Al, Matos DL. Tendências nas condições de saúde e uso de serviços de saúde entre idosos brasileiros: um estudo baseado na Pesquisa Nacional por Amostra de Domicílios: 1998-2003. Cad. Saúde Pública. 2007; 23(10):2467-78.

5.The WHOQOL Group. The World Health Organization quality of life assessment: position paper from the world health organization. Soc Sci Med. 1995; 41(10): 1403-09.

6.Virtuoso Junior IS, Guerra RO. Fatores associados às limitações funcionais em idosas de baixa renda. Rev Assoc Med Bras 2008; 54(5): 430-35.

7.Pedrazzi EC, Rodrigues RAP, Schiaveto FV. Morbidade referida e capacidade funcional de idosos. Cienc Saude Colet. 2007; 6(4): 407-13.

8.Lebrão ML. SABE - saúde, bem-estar e envelhecimento. 0 Projeto Sabe no município de São Paulo: uma abordagem inicial. Brasilia (DF): OPAS; 2003.

9. Ramos LR, Rosa TEC, Oliveira ZM, Medina MCG, Santos FRG. Perfil do idoso em área metropolitana na região sudeste do Brasil: resultados de inquérito domiciliar. Rev Saude Publica. 1987; 27(2): 87-94

10.Fleck MPA, Lousada S, Xavier M, Chachamovich E, Vieira G, Santos $L$, et al. Aplicação da versão em português do instrumento abreviado de avaliação da qualidade de vida WHOQOL-Bref. Rev Saude Publica. 2000; 34(2): 178-83.

11.Fleck MP, Chachamovich E, Trentini C. Development and validation of the Portuguese version of the WHOQOL-Old module. Rev Saude Publica.2006;40(5):785-91.

12.Sousa Al, Silver LD. Perfil sociodemográfico e estado de saúde auto-referido entre idosas de uma localidade de baixa renda. Esc Anna Nery. 2008; 12 (4): 706-16.

13. Camarano AA, Kansos S, Mello JL. Como vive o idoso brasileiro? In: Os novos idosos brasileiros: muito além dos 60 ? Rio de Janeiro (RJ): Ipea; 2004.p.25-73.

14.Fiedler MM, Peres KG. Capacidade funcional e fatores associados em idosos do sul do Brasil: um estudo de base populacional. Cad Saude Publica. 2008; 24(2): 409-15. 
15.Lima MG, Barros MBA, César CLG, Goldbaum M, Carandina L, Ciconelli RM. Health related quality of life among the elderly: a population-based study using SF-36 survey. Cad Saude Publica. 2009; 25(10): 2159-67.

16.Celich KLS. Domínios de qualidade de vida e capacidade para a tomada de decisão em idosos participantes de grupos de terceira idade [tese]. Porto Alegre: Pontifícia Universidade do Rio Grande do Sul; 2008.

\section{NOTA}

a Estudo decorrente de pesquisa financiada pela Fundação de Amparo à Pesquisa do Estado de Minas Gerais (FAPEMIG). 